\title{
Assessment of performance of services in a tertiary care Neuropsychiatric Institute using Pabon Lasso Model
}

\author{
Chandra Bhushan Tripathi', Rajesh Kumar', Ramesh Chandra Sharma ${ }^{3}$, Rachna Agarwal ${ }^{4}$ \\ ${ }^{1}$ Associate Professor, Department of Biostatistics, ${ }^{2}$ Associate Professor, Department of Psychiatry, ${ }^{3}$ Medical Record \\ Officer, Department of Medical Record, ${ }^{4}$ Associate Professor, Department of Neurochemistry, Institute of Human \\ Behavior \& Allied Sciences, New Delhi - 110 095, India
}

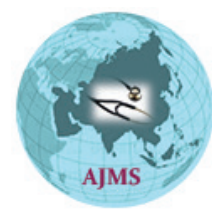

A B S T R A C T

Background: The objective of the study to assess optimal utilisation of hospital facilities \& evaluate their performance in a tertiary care hospital using Pabon Lasso Model using indicators- bed turnover (BTO), bed occupancy rate (BOR) and average length of stay (ALS). Aims and Objectives: To assess optimal utilisation of hospital facilities various wards catering to psychiatric, neurological and neurosurgery services in a tertiary care hospital using Pabon Lasso model and to identify strategies for more efficient use of the existing health service resources. Materials and Methods: This cross-sectional descriptive study was carried out in 2015 at the Institute of Human Behaviour and Allied Sciences, Delhi, India. This study involved various wards catering to psychiatric, neurological and neurosurgery facilities in the institute. Their performance was evaluated over 8 year period (2007-2014) using three performance indicators (BTO, BOR \& ALS) to assess optimal utilisation of hospital facilities. Results: Psychiatry department was initially located in quadrant IV in 2007 \& shifted to quadrant III in 2014 which suggests department's good quantitative performance with small proportion of unused beds. Similarly Neurosurgery department was in quadrant I at its inception in 2010, but shifted to quadrant III in 2014. However, Neurology department was located in quadrant III initially (2007), but shifted to quadrant II indicating either excess bed supply or less need for utilisation. Conclusion: Pabon Lasso model can be used by hospital management for evaluating the performance of health services in cost effective manner.

Key words: Performance indicators, Quality, Pabon Lasso model, BTO, BOR \& ALS
http://nepjol.info/index.php/AJMS DOI: 10.3126/ajms.v7i6.15408 E-ISSN: 2091-0576 P-ISSN: 2467-9100

\section{INTRODUCTION}

Increased life expectancy due to improved health services has led to increased aging population. This along with increase incidence of non communicable diseases has led to increase burden on diagnostic and treatment services. ${ }^{1}$ Government run hospitals/public hospitals, an essential component of health care system accounts for $50 \%$ spending on health services ${ }^{2,3}$ and constitute backbone of health system of developing countries. However, this increased pressure of health needs of general population leading to increased cost on diagnostic \& treatment facilities of health care system is putting immense pressure on overall quality of health care. ${ }^{4}$ To meet the increased demand for health services of high quality at cost effective level, health care providers have focussed not only on developing health facilities but also expansion of infrastructure i.e hospitals, laboratories etc. ${ }^{5}$ However, regular evaluation of performance of these hospitals/expanded health facilities is required to ensure the efficient use of these inputs not only in terms of utilisation of beds (inpatient days) and discharges but efficiency of economic measures such as average cost per inpatient day or per discharge. ${ }^{6}$ Such performance can prove to be an effective method for hospital management to use if evaluation is based on predetermined quality 
patient care plan on which hospital may be assessed to specify their effectiveness and efficiency ${ }^{7}$ rather than using traditional methods of trouble shooting/problem solving as and when they arise pertaining to personnel or system. Also, major challenges for policy makers of developing countries are technical and professional inefficiencies, failure to meet consumer/patient demands and lack of access for poorer sections of population.

Presently focus is largely on developing different models for evaluating the performance of health facilities/services for assessing efficiency of predetermined plans. One of the models that have been proved to be one of the most useful models for comparing the performance of different hospitals or different wards within same hospital is Pabon Lasso model. ${ }^{5,8}$

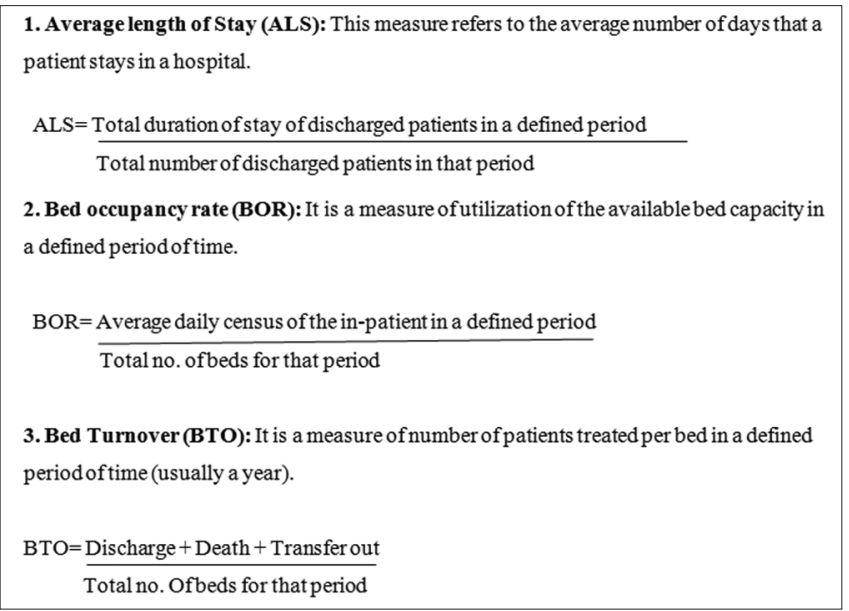

Figure 1: Performance indicators commonly used by the hospitals

\section{PABON LASSO MODEL}

Pabon Lasso model evaluates the performance using efficiency indicators namely bed turnover (BTO), bed occupancy rate (BOR) and average length of stay (ALS) ${ }^{5}$ (Figure 1) which we can use for achieving important goals of policy making, planning and resource management. ${ }^{5}$ Pabon Lasso graph divide hospitals into 4 categories based on these indicators (Figure 2):

Zone 1: Hospitals with low BTO and low BOR indicating a surplus of hospital beds relative to the existing demand.

Zone 2: Hospitals with high BTO and low BOR are characterised by unnecessary hospitalisation, an oversupply of beds or the use of beds for simply observing patients.

Zone 3: Hospitals with high BTO and high BOR have reached an appropriate level of efficiency with relatively few vacant beds at any time.

Zone 4: Hospitals with low BTO and high BOR are either serving patients with serious illnesses or have an unnecessary long ALS.

At present, development of health care services in India has been of predominantly quantitative in nature i.e expanding the health delivery system, without any serious assessment for it's efficiency. Pabon Lasso model can be applied for identification of poorly performing hospitals/ wards and in developing appropriate strategies to correct the inefficiencies. ${ }^{9-11}$ Such measures may also assist in transferring scarce resources to more cost effective interventions in the ambulatory care or primary care settings.

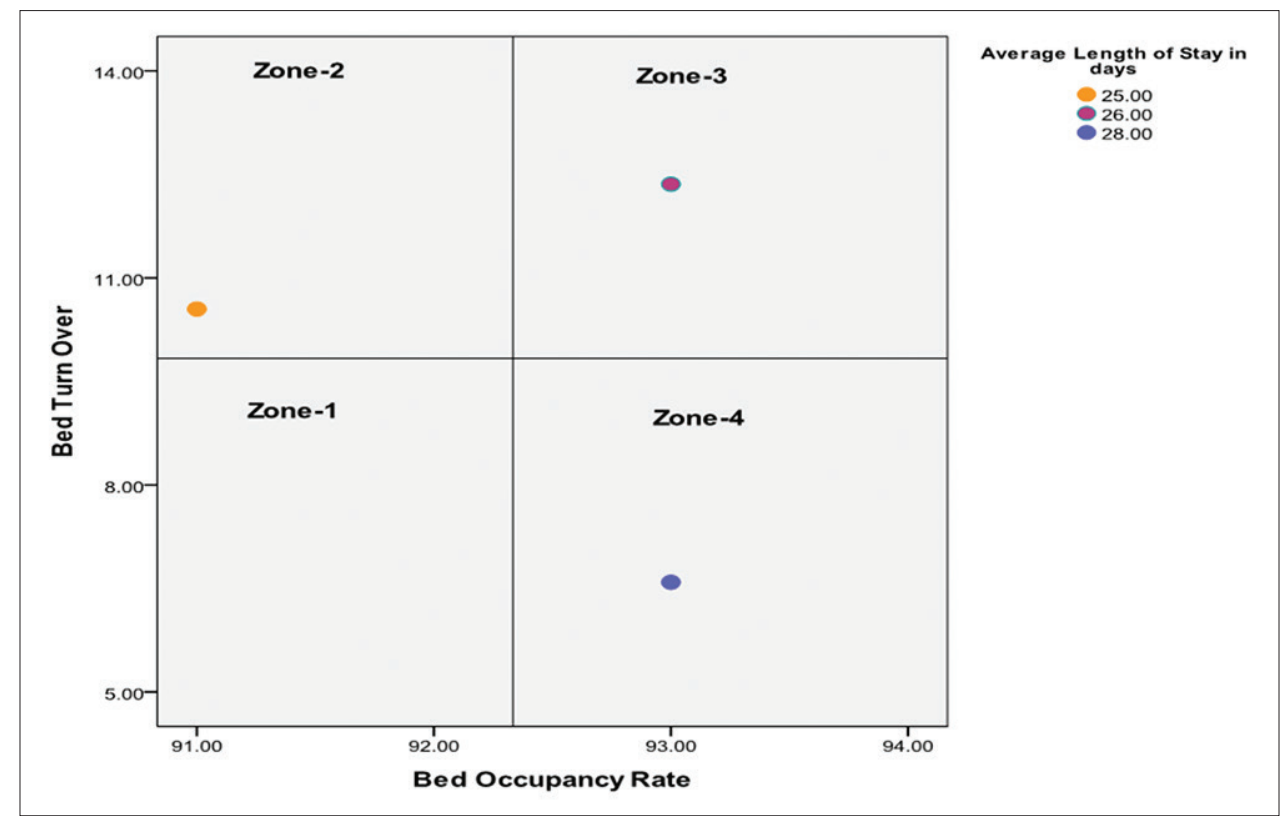

Figure 2: Pabon Lasso graph showing the performance status of psychiatry facility IHBAS 
Rational for the current study

Since its inception in April 1993, IHBAS has undergone major changes in terms infrastructure, quality of clinical services, patient care policies and related issues like investigations and treatment methods. IHBAS, being NABH accredited it is considered necessary and relevant to study the effect of all these changes on hospital performance and its efficiency. As we know, there is minimal budget allocation for health care in India more so for neuro-psychiatric disorder, hospitals have to be cost efficient and perform adequately even in the face of scarce resources. With this background, this study was planned and initiated to assess optimal utilisation of hospital wards catering to psychiatric, neurological and neurosurgery services in a tertiary care hospital using Pabon Lasso model and to identify strategies for more efficient use of the existing health service resources.

\section{MATERIAL AND METHODS}

This cross-sectional descriptive study was carried out in 2015 (data taken from 2007-2014) at the Institute of Human Behaviour and Allied Sciences (IHBAS), Dilshad Garden, Delhi, India. IHBAS, 346 bedded Neuropsychiatric tertiary care academic medical institution, located in northeastern part of Delhi, India.

\section{Data Collection}

Data was collected in two stages. In first stage questionnaire prepared in house was used to extract general data such as total number of beds in three disciplines, active bed-days, occupied bed-days, vacant bed-days, number of admissions, total number beds, number of discharges, number of deaths, number of transfer out, number of new admissions and leave against medical advice (LAMA cases). Data for this study was taken from computerized data bases (Source: data generated from the information obtained from filled questionnaires) in Medical Record Department (MRD) for it's three specialties- Psychiatry, Neurology, and Neurosurgery for $2007,2010 \& 2014$. In second stage, this data was stored in SPSS-18 and statistical analysis was performed to derive/calculate three performance indicators- BOR, BTO and ALS. These three indicators were the focus of present which were used to assess the performance of three specialities over the period of 8 years (from 2007-2014).

\section{Data analysis}

The data was analyzed using PASW ${ }^{@}$ Statistics -18 (SPSS18) software and Pabon Lasso graph.

\section{RESULTS}

The performance of various clinical departments (Psychiatry, Neurology \& Neurosurgery) of IHBAS over the period of 8 years (assessed in 2007, $2010 \& 2014$ ) has been summarized in Table 1. There were a total of 235 active hospital beds in 2007 which increased to 346 in 2013. Maximum active beds were in Psychiatry (195 in 2007 which increased to 270 in 2013), followed by Neurology (40 in 2007 to 54 in 2013) whereas Neurosurgery department became functional from 2010 with bed strength of 22). Total active bed days in IHBAS were highest in 2013 and 2014 (126290), out of which highest occupied bed days and vacant bed days were 126290 (in 2013 \& 2014) and 17809 (2013) respectively.

ALS of patients at IHBAS was 23 days in 2007, which decreased to 20 days in 2014. On an average, ALS of patients was highest in Psychiatry (25- 28 days) as compared to Neurology (7- 13 days) and Neurosurgery (11- 12 days). Similarly Bed Occupancy Rate (BOR) was highest in 2007 (92\%), which decreased to $87 \%$ in 2014. Interestingly, in Neurology BOR was high in 2007 (88\%), which gradually declined over the period of 8 years and was lowest in 2014 (74\%). However, BOR in Neurosurgery showed increase from $38 \%$ to $48 \%$ over the period of 4 years. According to obtained data, maximum Bed Turnover Rate (BTO) was 16.85 in 2014, as compared to BTO of 14.60 in 2010. Facility wise, maximum BTO was in Neurology (61.1 in 2007) and minimum BTO was in Psychiatry (6.59

Table 1: Performance of inpatient wards in psychiatry, neurology \& neurosurgery facilities of the hospital

\begin{tabular}{|c|c|c|c|c|c|c|c|c|c|c|c|c|}
\hline \multirow[t]{2}{*}{ Indicator } & \multicolumn{4}{|c|}{2007} & \multicolumn{4}{|c|}{2010} & \multicolumn{4}{|c|}{2014} \\
\hline & Psy & Neuro & Neusurg & Total & Psy & Neuro & Neusurg & Total & Psy & Neuro & Neusurg & Total \\
\hline ALS & 28 days & 7 days & -- & 23 days & 25 days & 13 days & 11 days & 20 days & 26 days & 12 days & 12 days & 20 days \\
\hline BOR & $93 \%$ & $88 \%$ & -- & $92 \%$ & $91 \%$ & $84 \%$ & $38 \%$ & $86 \%$ & $93 \%$ & $74 \%$ & $48 \%$ & $87 \%$ \\
\hline Turnover & 6.59 & 61.10 & -- & 15.87 & 10.55 & 31.35 & 18.23 & 14.60 & 12.36 & 38.31 & 19.23 & 16.85 \\
\hline Discharges & 1089 & 1911 & -- & 3000 & 1933 & 1219 & 310 & 3462 & 2287 & 1313 & 313 & 3913 \\
\hline Deaths & 14 & 56 & -- & 70 & 00 & 69 & 03 & 72 & 02 & 75 & 04 & 81 \\
\hline Transfer out & 182 & 477 & -- & 659 & 811 & 474 & 88 & 1373 & 1048 & 681 & 106 & 1835 \\
\hline New admission & 1082 & 1963 & -- & 3045 & 2009 & 1193 & 310 & 3512 & 2286 & 1300 & 306 & 3892 \\
\hline Total no. of beds & 195 & 40 & -- & 235 & 260 & 54 & 22 & 336 & 270 & 54 & 22 & 346 \\
\hline Active Bed-days & 71175 & 14600 & -- & 85775 & 94900 & 19710 & 8030 & 122640 & 98550 & 19710 & 8030 & 126290 \\
\hline $\begin{array}{l}\text { Occupied } \\
\text { Bed-days }\end{array}$ & 66384 & 12834 & -- & 79218 & 85983 & 16562 & 3078 & 105623 & 91708 & 14653 & 3858 & 110219 \\
\hline Vacant Bed-days & 4791 & 1766 & -- & 6557 & 8917 & 3148 & 4952 & 17017 & 6842 & 5057 & 4172 & 16071 \\
\hline
\end{tabular}


in 2007). However, in Psychiatry BTO improved in 2014 with 12.36, whereas it decreased in Neurology (38.3).

As one of these three indicators (ALS, BTO \& BOR) individuals may not give a comprehensive picture of department's performance, all these three indicators were used simultaneously in Pabon Lasso graph (Figures 2-4). Vertical and horizontal lines were set at the mean values of BOR \& BTO thereby forming four zones in Pabon Lasso graph. All three facilities i.e Psychiatry, Neurology and Neurosurgery were plotted in these four zones based on their performance indicators in 2007, 2010 and 2014. As shown in Figure 2, Psychiatric facilities were initially located in zone 4 in 2007 i.e they had high BOR with low BTO indicating either high proportion of long term cases or unnecessary long stay of patients. However, in
2014, Psychiatry facilities shifted in zone 3 having high BOR with high BTO. This observation suggests its good quantitative performance with small proportion of unused beds. Neurology facilities (Figure 3), initially (2007), was located in zone 3. Later with increase in bed strength, it shifted to zone 1 i.e low BOR and BTO. Performance of Neurosurgery facilities is shown in Figure 4. At it's inception (2010) it was located in zone 1 and shifted to zone 3 in 2014. This signifies improved quantitative performance with period of time.

\section{DISCUSSION}

This study was conducted using data from three clinical departments of IHBAS. As per our existing knowledge there has not been any such study reported in India till date.

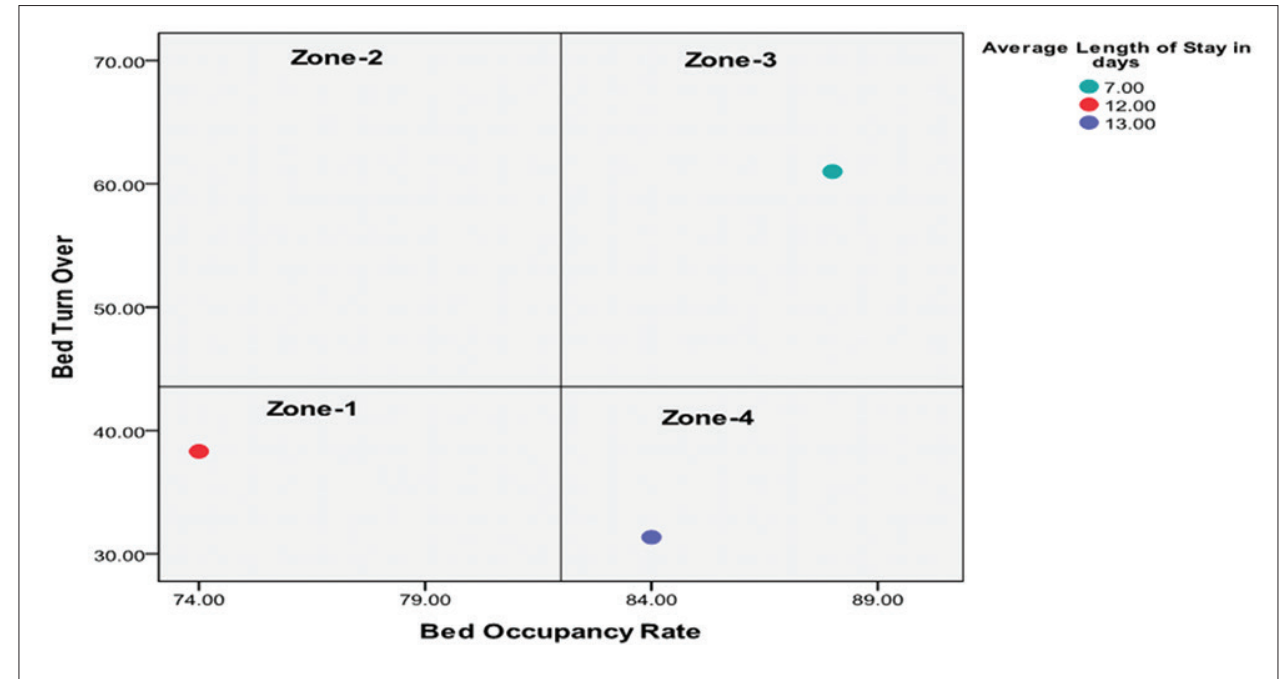

Figure 3: Pabon Lasso graph showing the performance status of neurological facility in IHBAS

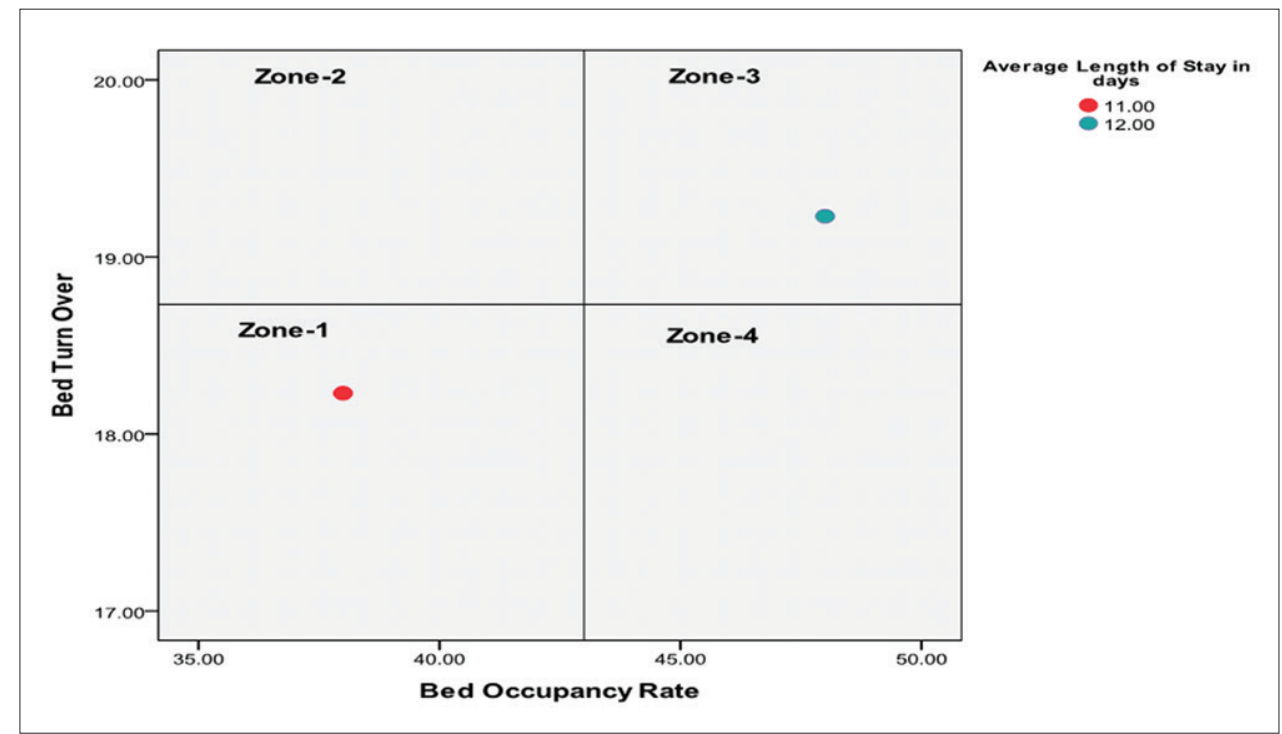

Figure 3: Pabon Lasso graph showing the performance status of neurosurgical facility in IHBAS 
Some studies performed previously examined one or two performance indicators in piecemeal such as bed utilisation indices, ${ }^{12} \mathrm{BOR}$ and ALS. ${ }^{13}$ Although single indicator is easy to use but may lead to misleading results. ${ }^{14}$ For example, high BOR may not necessarily indicate optimal utilisation of beds which signifies good performance, rather may indicate long term unnecessary hospitalisation of patient or poor nursing care, delayed diagnostic and therapeutic interventions or development of hospital acquired infections. ${ }^{14}$ Therefore to avoid such erroneous conclusions arising from use of single performance indicators, Pabon Lasso model has been applied using three main performance indicators (ALS, BOR \& BTO) in this study to assess optimal utilisation of hospital facilities in various wards catering to psychiatric, neurological and neurosurgery health facilities at IHBAS over the period of 8 years (2007-2014).

In the present study, ALS declined from 23 days to 20 days over the period of 8 years. In 2007, the longest length of stay was recorded for Psychiatry with an average of 28 days, while the lowest was for the Neurology with an average of 7 days. In India, Reddy et a ${ }^{15}$ reported ALS of 20.9 days for psychiatric patients in teaching hospitals, whereas Ravi Kiran $\mathrm{E}$ et $\mathrm{al}^{16}$ found an ALS of 9 days in a tertiary care teaching hospital. The length of stay of patients was highest in psychiatry department as most of the mental illnesses are recurrent and chronic in nature. IHBAS being tertiary care neuro-psychiatric institute usually receives treatment refractory cases that require long term hospitalization. IHBAS also receives most of the court referred cases where patients are homeless mentally ill without family and these cases usually stay in the ward for longer period in view of the problems in assessment, management and social rehabilitation. These factors usually make the ALS longer. After lots of collective efforts from the various professionals, we have been able to reduce the ALS in psychiatry. Mostly efforts had been made to increase BTO by upgrading the diagnostic facilities viz a viz imaging and laboratory facilities, in addition to improved resources in terms of staff both nursing and medical doctors, increased strength of beds.

The BOR for any hospital indicates utilisation of available bed strength and hospital's operating efficiency. In present study the bed occupancy for the hospital was near optimal throughout the study period (87-92\%). However, there was a fall in BOR in Neurology in 2014 (80\%). Also, Neurosurgery had lowest BOR, though there was a marginal increase in BOR from $38 \%$ in 2010 to $48 \%$ in 2014 indicating under utilisation of beds. It may be a strong case for decreasing the bed strength which could increase its operational efficiency. Barnum et $\mathrm{a}^{17}$ suggested in their study that the hospitals would be operating maximally at an occupancy rate of $85-90 \%$. In an Indian study Anand TR ${ }^{18}$ has also reported an occupancy rate of $80-90 \%$ as optimal.
The BTO of a hospital is an important hospital utilisation and is inversely related to ALS. It indicates that hospital is operating at a high level of efficiency vis a vis bed utilisation. ${ }^{12}$ Too large a turnover rate indicates that only simple first aid types of treatments are being provided by the hospital. Too small a turnover rate would indicate fewer people utilizing the hospital and patients being retained unnecessarily. However it is expected to be higher in acute care hospitals than chronic care hospitals. ${ }^{19}$ In our study, BTO was 14.60 days in 2010 which increased to 16.85 days in 2014. Department wise minimal BTO was lowest in Psychiatry (6.59 in 2007) which increased in 2014 to 12.36. This overall increase in BTO was due to improved neuropsychiatric investigation facilities like neurophysiological lab, imaging facilities- MRI, CT Scan \& Laboratory diagnostic facilities. This led to early diagnosis followed by appropriate intervention in the patient. Hence, it prevented unnecessary and undue stay of patient in the hospital. However, BTO in Neurology (61.1 in 2007) decreased to 38.31 in 2014. It is difficult to conclude that there are excess beds in Neurology along with low demand/utilisation, but in last few years there has been drastic decrease in number of consultants. Indian literature available on BTO studied in tertiary care hospitals shows mixed results. Vaz et $\mathrm{al}^{12}$ has reported bed turnover rate of 55 in 2006 in Goa Medical College Hospital in India which is a tertiary care hospital, ${ }^{12}$ whereas Ravi Kiran et a ${ }^{16}$ reported a turnover rate of 7.1 days in a tertiary hospital. We could not find any such study done in Psychiatry set up.

When all three clinical facilities (Psychiatry, Neurology \& Neurosurgery) were compared on the basis of their position in the four zones of Pabon Lasso graph, plotted using three hospital performance indicators (BTO, BOR and ALS) Psychiatric services were initially located in zone 4 in 2007 (High BTO and BOR, characteristic of long term inpatient facilities such as psychiatric and geriatric medicine) and shifted to zone 3 in 2014. This suggests that wards catering to psychiatry \& neurosurgery services have good quantitative performance with small proportion of unused beds. Also, such shift to zone 3 indicates not only good efficiency of these services, but also having experienced medical, paramedical and administrative staff, availability of advanced medical facilities including equipments and technology. ${ }^{4}$ However, Neurology services were located in zone 3 initially (2007), but shifted to zone 1 in 2014. Though it can be argued that low BOR indicates that patients are being admitted unnecessarily or lots of beds are lying vacant for long periods of time, but with low BTO, characteristic of low demand/utilisation, it can be converted to an efficient facility by avoiding unnecessary or prolonged admission. ${ }^{4}$ However, study need to be conducted to identify the factors contributing to underutilisation of beds, as vacant beds don't necessarily imply that presence of excess capacity relative to 
need. This may help in instituting appropriate measures that will enhance optimal use of existing hospital bed capacity. ${ }^{14}$

Pabon Lasso model can be used for identification of the hospitals with poor performances and highlight areas to direct rectification of their inefficiencies, but a number of other factors such as access to communication facilities, lack of availability of home or community care, teaching hospital status, the number of employees and hospital facilities cannot be measured by it. ${ }^{4}$ However, visual position of various hospitals or different wards in same hospital on the Pabon Lasso graph facilitates the understanding of the relationship between various performance indicators and the efficient use of resources and can enable the planners to make appropriate and scientific decisions about hospitals. ${ }^{5}$ As the Pabon Lasso model is a valuable tool that is easy to use, it is recommended that hospital management must conduct this kind of analysis to check effectiveness of various changes in hospital policy and thereby provide evidence to health care planners and policy makers for facilitating the management decision making processes improvement plans and provide evidence for management decision-making purposes.

\section{REFERENCES}

1. Mohebbifar R, Sokhanvar M, Hassanpoor E, Isfahani HM, Ziaiifar H, Kakemam E and Mohseni M. A Survey on the Performance of Hospitals of Qazvin Province by the Pabon Lasso Model. Int Res J Biological Sci 2014;3(12):5-9.

2. Mills $A$ and Colclough $C$. Improving the efficiency of public sector health services in developing countries: Bureaucratic versus market approaches, marketizing education and health in developing countries: Miracle or mirage 1997;245-274.

3. Hanson K, Atuyambe L, Kamwanga J, McPake B, Mungule O and Ssengooba F. Towards improving hospital performance in Uganda and Zambia: Reflections and opportunities for autonomy. Health policy 2002;61(1):73-94.

4. Davoud A, Issac BG. Sadeghi, Mohammad P, Hossein A, Salarkhah E. Contemporary use of hospital efficiency indicators to evaluate hospital performance using the Pabon Lasso model. European J Buss \& Soci Sci 2014;3(2):1-8.
5. GoshtasebiA, Vahdaninia M, Gorgipour R, SamanpourA, Maftoon F, Farzadi F and Ahmadi F. Assessing Hospital Performance by the Pabon Lasso Model. Iranian J Publ Health 2009;38(2):119-124.

6. Use of the indicators to assess hospital efficiency. KwaZulaNatal Epidemiology Bulletin, Published by The Epidemiology Unit, KwaZula-Natal Department of Health, Private Bag X9051, Pietermaritzburg 3200. 2004;7:6-34.

7. Asefzade S. Health Economics Principles, Ghazvin: Hadise emrouz Publications 2003.

8. Pabón Lasso H. Evaluating hospital performance through simultaneous application of several indicators. Bulletin of the Pan American Health Organization 1986;20 (4):341-357.

9. Jakab M, Harding A, Preker A, Hawkins L. Organizational reform and management of public providers: focus on hospitals: appendix 3: critical skills: monitoring and evaluating hospital performance. Washington D. C, World Bank, 2000.

10. Forster DP, Frost B, Kamberska Z, Holub J. Supply, utilisation and outcome in hospital systems: an Anglo-Czech comparison. Health policy 1999;48:171-187.

11. Peyravi H, Neyati J, Afshar M, Emami H, Pazargadi M. The role of operational criteria in determination of objectives and decision-making strategies in Universities of Medical Sciences and Health Services. Pejouhandeh Quaterly Research Journal 2000;19:241-254.

12. Vaz FS, Ferreira AM, Kulkarni MS and Motghare DD. Bed Utilization Indices at a Tertiary Care Hospital in Goa: An Eight Year Trend Analysis. Indian J Public Health 2007;51(4): 231-233.

13. Singh GP, Chavan BS, Arun P, Sidana A. Seasonal pattern of psychiatry service utilization in a tertiary care hospital. Indian $\mathrm{J}$ Psychiatry 2007;49(20):91- 95.

14. WHO. The African Health Monitor: Health Systems and Reproductive Health, Special Issue 14. WHO, Geneva, 2006.

15. Reddy MV, Kaliaperumal VG, Channabasavanna SM. Mental health delivery system in general hospitals attached to medical colleges. Indian J Psychiatry 1995;37:176-178.

16. Ravi KE, Vijaya K. Utilization of beds in a tertiary hospital. Journal of Association of Hospital Administrators 2004;15(2):13-17.

17. Barnum H, Kutzin J. Public Hospitals in Developing Countries: Resource use, Cost, Financing. Johns Hopkins University Press for the World Bank, Baltimore, 1993.

18. Anand TR. Hospital services and management methods, Background reading material for training course in Hospital management, New Delhi, 1992.

19. Dhungan HN, Siddiqui MU, Sahu KK. Role of Hospital Utilization Constants as Past Performing and Future Planning Markers. Asian Journal of Management Sciences 2014;02 (05):01-04.

\section{Authors Contribution:}

CBT - Concept, literature search, statistically analysed and interpreted and review of study; RK - Concept of study, helped in preparing first draft of manuscript and critical revision of the manuscript; RCS - Collecting data; RA - Conceptualized study and design of the study, reviewed the literature, manuscript preparation and critical revision of the manuscript.

Source of Support: Nil, Conflict of Interest: None declared. 